\title{
Evolution to the equilibrium in a dissipative and time dependent billiard
}

\author{
Marcus Vinícius Camillo Galia a ${ }^{a}$ Diego F.M. Oliveira ${ }^{\mathrm{b}, c, \mathrm{~d}, *}$, Edson D. Leonel $^{\mathrm{a}, \mathrm{e}}$ \\ ${ }^{a}$ Departamento de Física, UNESP, Univ. Estadual Paulista, Av. 24A, 1515, Bela Vista, 13506-900, Rio Claro, SP, Brazil \\ ${ }^{\mathrm{b}}$ Center for Complex Networks and Systems Research, School of Informatics and Computing, Indiana University, Bloomington, IN, USA \\ ${ }^{c}$ Department of Chemical and Biological Engineering, Northwestern University, Evanston, IL 60208, USA \\ ${ }^{\mathrm{d}}$ Northwestern Institute on Complex Systems (NICO), Northwestern University, Evanston, IL 60208, USA \\ ${ }^{e}$ Abdus Salam International Center for Theoretical Physics, Strada Costiera 11, 34151 Trieste, Italy
}

\section{A R T I C L E I N F O}

\section{Article history:}

Received 19 July 2016

Available online 11 August 2016

\section{Keywords:}

Billiards

Dissipations

Thermodynamic

\begin{abstract}
A B S T R A C T
We study the convergence towards the equilibrium for a dissipative and stochastic timedependent oval billiard. The dynamics of the system is described by using a generic four dimensional nonlinear map for the variables: the angular position of the particle, the angle formed by the trajectory of the particle with the tangent line at the position of the collision, the absolute velocity of the particle, and the instant of the hit with the boundary. The dynamics of the stationary state as well as the dynamical evolution towards the equilibrium is made by using an ensemble of non interacting particles. Finally, we make a connection with the thermodynamic by using the energy equipartition theorem.
\end{abstract}

(C) 2016 Elsevier B.V. All rights reserved.

\section{Introduction}

Classical billiards are dynamical system in which a particle, or an ensemble of non-interacting particles, moves confined to and experiences collisions with a boundary [1-9]. Basically, they are settled in three classes, namely (i) integrable, (ii) ergodic, and (iii) mixed. In case (i), the phase space consists of invariant tori filling the entire phase space. In case (ii), the time evolution of a single initial condition is enough to fills up the entire phase space. Finally, in case (iii), one can observe invariant tori, chaotic seas generally surrounding Kolmogorov-Arnold-Moser (KAM) coexisting. If a time dependent perturbation is introduced on the boundary [10], the system exchanges energy with the moving particles upon collisions. Such type of systems have attracted a lot of attention lately because they can be used to study the phenomenon of unlimited energy growth also known as Fermi acceleration [11]. However, how to identify in which type of system the phenomenon of Fermi acceleration will be observed? To answer this question, Loskutov, Ryabov and Akinshin (LRA) proposed a conjecture where they state that a chaotic component in the phase space for the time-independent dynamics is a sufficient condition to observe Fermi acceleration once a time dependent perturbation on the boundary is introduced. This conjecture became known as LRA conjecture [12,13] and over the years it has been verified in several systems such as the time dependent Lorentz gas [14,15], oval [16] and stadium [17,18] billiard among many other systems [19,20]. Nevertheless, later on results have shown that the existence of a chaotic component is a sufficient, but not a necessary condition for Fermi acceleration since the unlimited energy growth was also observed in a time dependent elliptical billiard. As it is known, the elliptical with static boundary is an integrable system whose integrability comes from the conservation of the angular momentum with

\footnotetext{
* Corresponding author at: Department of Chemical and Biological Engineering, Northwestern University, Evanston, IL 60208, USA.

E-mail address: diegofregolente@gmail.com (D.F.M. Oliveira).
} 


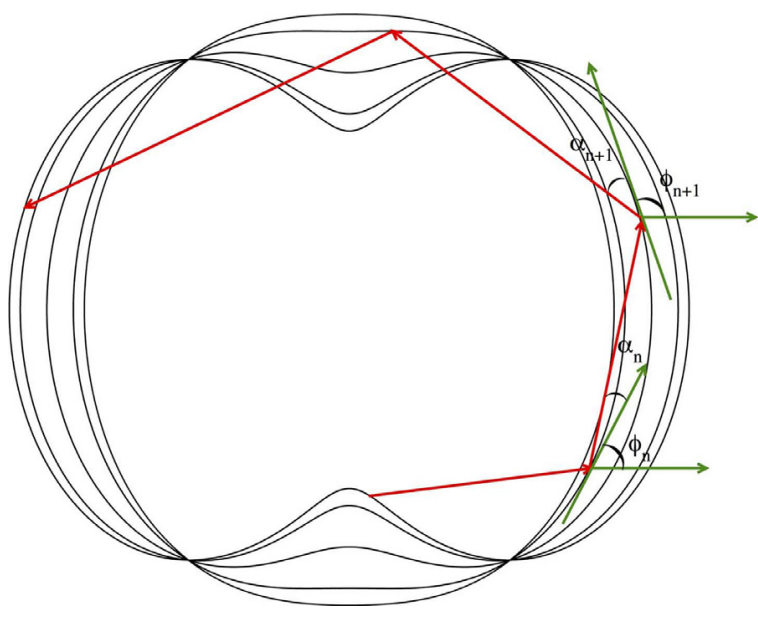

Fig. 1. Illustration of 5 snapshots of a time-dependent oval billiard. The corresponding angles that describe the dynamics of the model are also shown for two collisions.

respect to the two foci [21]. However, when a time dependent perturbation is introduced into the system, the separatrix is replaced by a chaotic layer and trajectories that were confined inside the separatrix (librators) can now explore the region outside the separatrix (rotators) and vice-versa. This change of behavior, namely, librator orbits "jumping" to rotator and vice-versa turned out to be the mechanism which produces the unlimited energy growth [22-24]. More recently, it became clear that such a phenomenon is not robust [25] since a tiny amount of dissipation, either upon collision [26,27] or during the flight [28], is enough to suppress Fermi acceleration.

The motion of the time dependent boundaries can be related to a more physical situation. Indeed due to the thermal fluctuations, the position of each atom on the boundary is allowed to move locally. Such oscillation of the atoms, and hence of the boundary, can be extended to the context of billiard which allows us to connect the observables obtained from the velocity of the particle - such as the kinetic energy - to the thermodynamics, more precisely, the temperature and entropy [29]. So far, such a connection has been made for the Lorentz gas to describe the motion of electrons between heavy ions as in a lattice of metal [30]. Therefore, further investigation is needed to understand how the dynamics of the systems can be connected to more real situations.

In this paper we study some dynamical properties for an ensemble of noninteracting particles in a time dependent billiard. Our main goal is to understand and describe the dynamics of the mean squared velocity as a function of the time considering different values of the control parameters, namely, the dissipation upon collision, the parameter that controls the shape of the boundary and the amplitude of the time-dependent perturbation of particles moving inside a closed domain. As an illustration, we consider a stochastic version of the time-dependent oval billiard. The introduction of a random perturbation on the border resembles the rough oscillations - producing a random exchange of energy upon collision - at least in the microscopic domain. The results that are obtained numerically are confirmed by using an analytical approach.

This paper is organized as follows. In Section 2 we present the model. In Section 3 we present our numerical findings. In Section 4 we introduce an analytical approach to obtain the behavior of the average velocity as a function of the number of collisions with the moving boundary. Finally, in Section 5, we make a connection with the thermodynamics by using the expression obtained analytically for the particle's average velocity. Conclusions are drawn in Section 6.

\section{The model and the map}

In this section we present all the details needed to study the dynamics of an ensemble of noninteracting particles experiencing collisions with a moving boundary. As it is so usual in the literature, we describe the dynamics of the model in terms of a four dimensional nonlinear mapping $\tilde{T}$ that gives the angular position of the particle $\theta_{n}$; the angle that the trajectory of the particle forms with the tangent line at the position of the collision $\alpha_{n}$; the absolute velocity of the particle $\left|\vec{V}_{n}\right|$ and the instant of the hit with the boundary $t_{n}$, i.e., $\left(\theta_{n+1}, \alpha_{n+1},\left|\vec{V}_{n+1}\right|, t_{n+1}\right)=\tilde{T}\left(\theta_{n}, \alpha_{n},\left|\vec{V}_{n}\right|, t_{n}\right)$. The index $n$ denotes the $n$th collision with the moving boundary. Assuming that the shape of the boundary in polar coordinates is $R_{b}(\theta, \epsilon, p, t)=1+\varepsilon f(t) \cos (p \theta)$ where the subindex $b$ denotes boundary, $f(t)$ is a function to be chosen, $\varepsilon$ is the perturbation of the circular billiard and $p$ is an integer number warranting a closed boundary, otherwise particles would escape. We consider $f(t)=1+\eta \cos (\omega t)$ where $\eta$ is the amplitude of the time dependent perturbation and $\omega$ is the angular frequency, which from now is fixed as $\omega=1$. Fig. 1 shows a typical illustration of a billiard and the angles used to describe the dynamics of the model. 
For a given state $\left(\theta_{n}, \alpha_{n},\left|\vec{V}_{n}\right|, t_{n}\right)$, the position of the particle as a function of the time is written as

$$
\begin{aligned}
& X(t)=X\left(\theta_{n}, t_{n}\right)+\left|\vec{V}_{n}\right| \cos \left(\alpha_{n}+\phi_{n}\right)\left(t-t_{n}\right), \\
& Y(t)=Y\left(\theta_{n}, t_{n}\right)+\left|\vec{V}_{n}\right| \sin \left(\alpha_{n}+\phi_{n}\right)\left(t-t_{n}\right),
\end{aligned}
$$

with $t \geq t_{n}$ and $X\left(\theta_{n}, t_{n}\right)=R_{b}\left(\theta_{n}, t_{n}\right) \cos \left(\theta_{n}\right)$ and $Y\left(\theta_{n}, t_{n}\right)=R_{b}\left(\theta_{n}, t_{n}\right) \sin \left(\theta_{n}\right)$. Furthermore, once the angular position $\theta$ is known, the angle between the tangent line and the horizontal at $X(\theta), Y(\theta)$ is $\phi=\arctan \left[Y^{\prime}(\theta, t) / X^{\prime}(\theta, t)\right]$ where $Y^{\prime}(\theta, t)=\mathrm{d} Y / \mathrm{d} \theta$ and $X^{\prime}(\theta, t)=\mathrm{d} X / \mathrm{d} \theta$.

Once the particle hits the moving boundary it is reflected and travels with a constant speed until the particle experiences the collision $(n+1)$. The distance traveled by the particle measured with respect to the origin of the coordinate system is given by $R(t)=\sqrt{X^{2}(t)+Y^{2}(t)}$. Therefore, the angular position at the next collision of the particle with the boundary, $\theta_{n+1}$, is obtained by solving the equation $R\left(\theta_{n+1}, t_{n+1}\right)=R_{b}\left(\theta_{n+1}, t_{n+1}\right)$. This means that the position of the particle is the same as the boundary, hence producing a collision. Moreover, we can also obtain the time at collision $n+1$ by evaluating the expression

$$
t_{n+1}=t_{n}+\frac{\sqrt{\Delta X^{2}+\Delta Y^{2}}}{\left|\vec{V}_{n}\right|}+Z(n),
$$

where $\Delta X=X_{p}\left(\theta_{n+1}, t_{n+1}\right)-X\left(\theta_{n}, t_{n}\right)$ and $\Delta Y=Y_{p}\left(\theta_{n+1}, t_{n+1}\right)-Y\left(\theta_{n}, t_{n}\right)$ and $Z(n)$ is a number taken at random from the interval $[0,2 \pi]$ introduced after the instant of collision and it is the variable that introduces stochasticity into the model. To obtain the particle's velocity at time $t_{n+1}$, we should note that the referential frame of the boundary is moving. Furthermore, we assume the particle experiences a fractional loss of velocity/energy upon collision only on the normal component of the velocity. Therefore, at the instant of collision, the following conditions must be obeyed

$$
\begin{aligned}
& \vec{V}_{n+1}^{\prime} \cdot \vec{T}_{n+1}=\vec{V}_{n}^{\prime} \cdot \vec{T}_{n+1}, \\
& \vec{V}_{n+1}^{\prime} \cdot \vec{N}_{n+1}=-\gamma \vec{V}_{n}^{\prime} \cdot \vec{N}_{n+1},
\end{aligned}
$$

where the unit tangent and normal vectors are

$$
\begin{aligned}
& \vec{T}_{n+1}=\cos \left(\phi_{n+1}\right) \hat{i}+\sin \left(\phi_{n+1}\right) \hat{j}, \\
& \vec{N}_{n+1}=-\sin \left(\phi_{n+1}\right) \hat{i}+\cos \left(\phi_{n+1}\right) \hat{j} .
\end{aligned}
$$

The parameter $\gamma \in[0,1]$ is the restitution coefficient. Thus, if $\gamma=1$ we have the case of completely elastic collisions while $\gamma<1$ leads the particle to have partial loss of velocity/energy upon collisions. The term $\vec{V}^{\prime}$ corresponds to the velocity of the particle in the non-inertial reference frame. Using the equations above, one can find that the tangential and normal components of the velocity after collision $n+1$ are given by

$$
\begin{aligned}
& \vec{V}_{n+1} \cdot \vec{T}_{n+1}=\vec{V}_{n} \cdot \vec{T}_{n+1}, \\
& \vec{V}_{n+1} \cdot \vec{N}_{n+1}=-\gamma \vec{V}_{n} \cdot \vec{N}_{n+1}+(1+\gamma) \vec{V}_{b}\left(t_{n+1}\right) \cdot \vec{N}_{n+1},
\end{aligned}
$$

where $\vec{V}_{b}\left(t_{n+1}\right)$ is the velocity of the boundary and it is written as

$$
\vec{V}_{b}\left(t_{n+1}\right)=\left.\frac{\mathrm{d} R(t)}{\mathrm{d} t}\right|_{t_{n+1}}\left[\cos \left(\theta_{n+1}\right) \widehat{i}+\sin \left(\theta_{n+1}\right) \widehat{j}\right] .
$$

Finally, the velocity of the particle after the collision $(n+1)$ is given by

$$
\left|\vec{V}_{n+1}\right|=\sqrt{\left(\vec{V}_{n+1} \cdot \vec{T}_{n+1}\right)^{2}+\left(\vec{V}_{n+1} \cdot \vec{N}_{n+1}\right)^{2}}
$$

and $\alpha_{n+1}$ is written as

$$
\alpha_{n+1}=\arctan \left[\frac{\vec{V}_{n+1} \cdot \vec{N}_{n+1}}{\vec{V}_{n+1} \cdot \vec{T}_{n+1}}\right] .
$$

With the equation above, we have all the ingredients needed to study the dynamics of the system.

\section{Numerical results for the stochastic dynamics}

In this section we concentrate to characterize the behavior of the average velocity in terms of the number of collisions with the boundary and as a function of the control parameters $\gamma, \eta$ and $\varepsilon$. Here $\gamma$ is the dissipation parameter acting only along the normal component of the particle's velocity, $\varepsilon$ is the perturbation on the boundary and $\eta$ is the amplitude of the time perturbation. We consider a dissipative version of the oval-shaped billiard [26] close to the transition from unlimited 

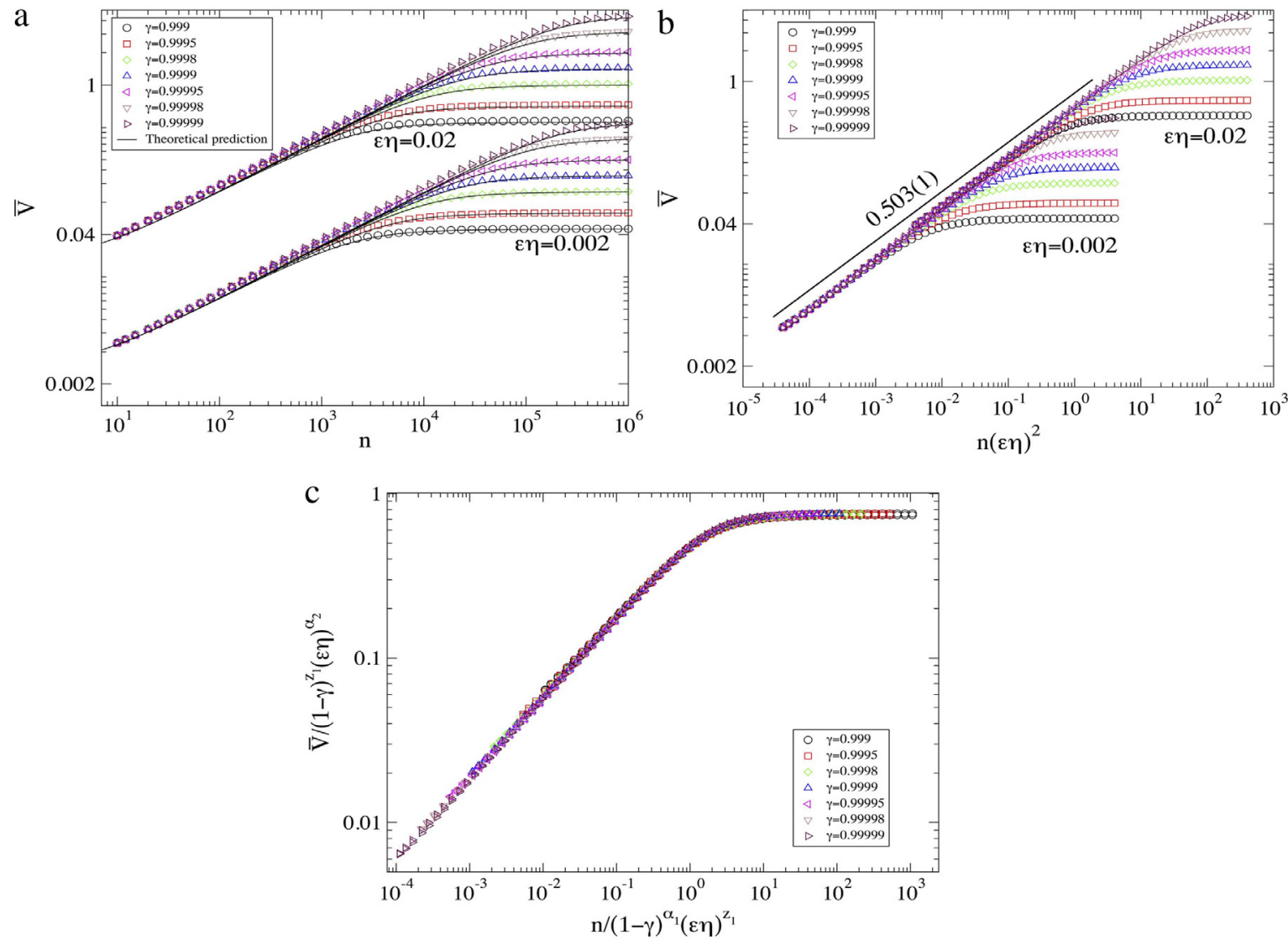

Fig. 2. (a) Behavior of the average velocity as a function of the number of collisions for different values of $\gamma$ and two different combinations of $\eta \varepsilon$. (b) Their initial collapse after the transformation $n \rightarrow n(\varepsilon \eta)^{2}$. (c) Overlap of all the curves of the average velocity onto a single and universal plot.

to limited energy growth. Such a transition happens when the control parameter $\gamma \rightarrow 1$. However, the transition is better characterized for $(1-\gamma)$. To obtain the average velocity, each initial condition has a fixed initial velocity, $V_{0}=10^{-3}$, $\eta \varepsilon \in[0.002,0.02]$ and randomly choose $\alpha_{0} \in[0, \pi], \theta_{0} \in[0,2 \pi], t_{0} \in[0,2 \pi]$. In addition, after every time step, a random number $[Z(n)]$ is introduced in Eq. (3), thus, introducing stochasticity into the model. Two different procedures were applied in order to obtain the average velocity. Firstly, we evaluate the average velocity over the orbit for a single initial condition and then over an ensemble of initial conditions. Hence, the average velocity is written as

$$
\bar{V}=\frac{1}{M} \sum_{i=1}^{M} \frac{1}{n+1} \sum_{j=0}^{n} V_{i, j},
$$

where the index $i$ corresponds to a sample of an ensemble of initial conditions, $M$ denotes the number of different initial conditions. We have considered $M=2000$ in our simulations. The behavior of $\bar{V} v s . n$ for different values of $\gamma$ is shown in Fig. 2(a). It is easy to see in Fig. 2 two different kinds of behaviors. For short $n$, the average velocity grows according to a power law and suddenly it bends towards a regime of saturation for long enough values of $n$. The changeover from growth to the saturation is marked by a crossover iteration number $n_{x}$. Different values of $\eta \varepsilon$ generate different behaviors for the average velocity, however, applying the transformation $n \rightarrow n(\eta \varepsilon)^{2}$, all the curves start to grow together as shown in Fig. 2(b). For such a behavior, we propose the following scaling hypotheses:

1. For small values of $n$, such as $n \ll n_{x}$, the growth regime can be described by

$$
\bar{V} \propto\left[(\eta \varepsilon)^{2} n\right]^{\beta},
$$

where $\beta$ is the acceleration exponent.

2. For sufficiently large $n$, say $n \gg n_{x}$, where the regime of saturation is reached, we have

$$
V_{\text {sat }} \propto(1-\gamma)^{\alpha_{1}}(\eta \varepsilon)^{\alpha_{2}}
$$

where $\alpha_{1}$ and $\alpha_{2}$ are the saturation exponents. 

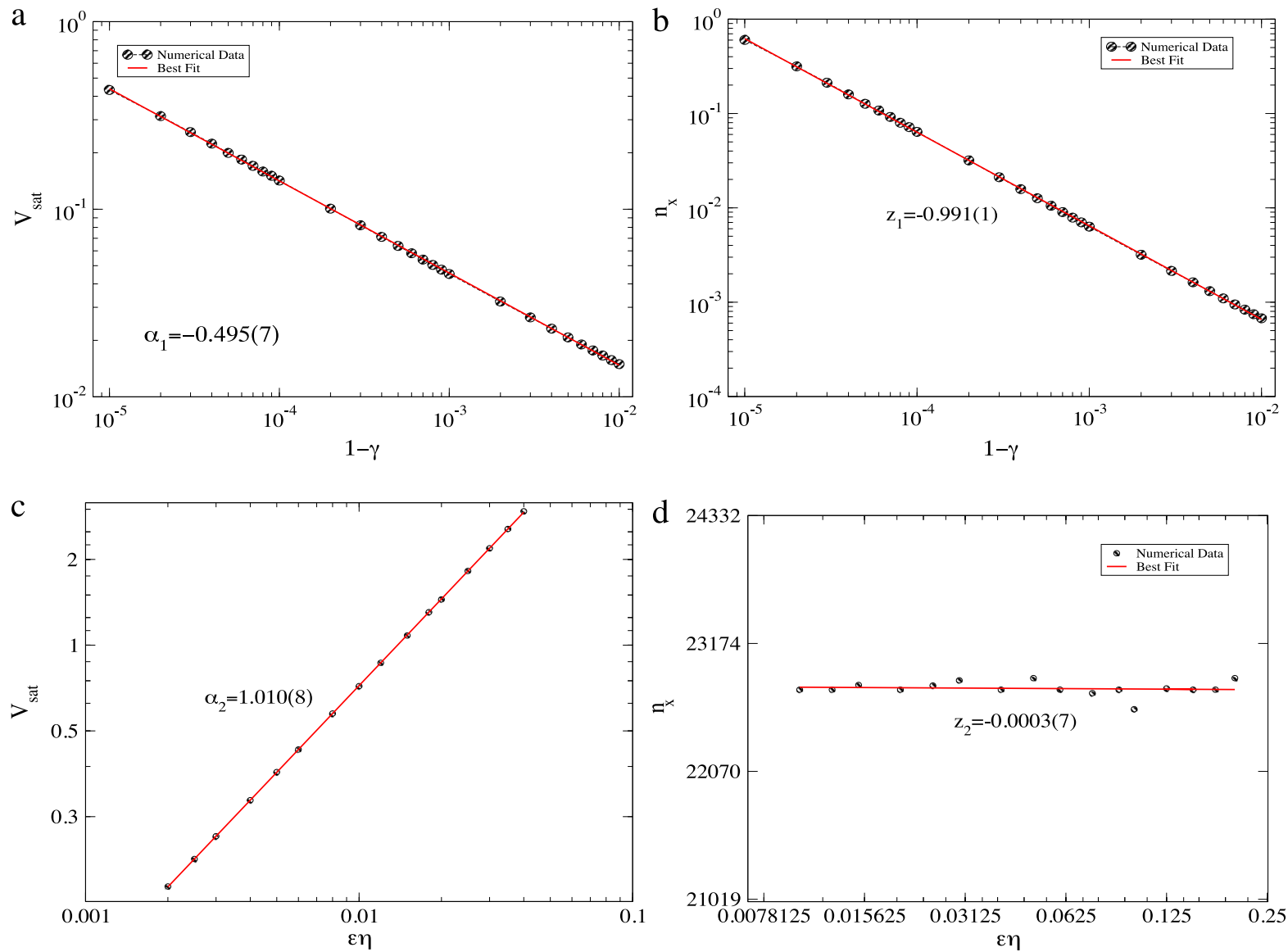

Fig. 3. Behavior of (a) $V_{\text {sat }}$ and (b) $n_{x}$ as a function of $(1-\gamma)$. The behavior of $V_{\text {sat }}$ and $n_{x}$ for different values of $\varepsilon \eta$ is shown in figures (c) and (d), respectively. Observe that $n_{x}$ does not depend on $\varepsilon \eta$, hence $z_{2}=0$.

3. Finally, the characteristic value of $n$ which provides the changeover from growth to the saturation is given by

$$
n_{x} \propto(1-\gamma)^{z_{1}}(\eta \varepsilon)^{z_{2}}
$$

where $z_{1}$ and $z_{2}$ are crossover exponents.

Once the scaling hypotheses are known, we propose that the behavior of the average velocity can be described by a homogeneous function of the type

$$
\bar{V}\left[(\eta \varepsilon)^{2} n, \eta \varepsilon,(1-\gamma)\right]=\operatorname{lV}\left[l^{b}(\eta \varepsilon)^{2} n, l^{c} \eta \varepsilon, l^{d}(1-\gamma)\right],
$$

where $l$ is a scale factor, $b, c$ and $d$ are characteristic exponents that in principle must be related to the scaling exponents. Since $l$ is a scaling faction, one can choose the quantities $l^{b}(\eta \varepsilon)^{2} n=1, l^{c} \eta \varepsilon=1$ and $l^{d}(1-\gamma)=1$ and after some calculation [31], it is straightforward to show that the scaling exponents are related by the following expressions

$$
z_{1}=\frac{\alpha_{1}}{\beta}, \quad z_{2}=\frac{\alpha_{2}}{\beta}-2
$$

Now, all we need is to find the exponents numerically. A power law fitting in $\bar{V}$ when $n \ll n_{x}$ gives us that $\beta=0.503(1) \simeq$ $1 / 2$. Such value was obtained from the range of $\gamma \in[0.999,0.99999]$. Considering $\eta \varepsilon$ fixed, after a power law fitting on the plot $V_{\text {sat }} v s$. $(1-\gamma)$ we obtained $\alpha_{1}=-0.495$ (7) (see Fig. 3(a)). The plot of $n_{x} v s$. $(1-\gamma)$ gives us $z_{1}=-0.991(1)$. On the other hand, if $(1-\gamma)$ is fixed, the behavior of $V_{\text {sat }} v s$. $\eta \varepsilon$ gives a slope $\alpha_{1}=1.010(8)$ while a plot of $n_{x} v s$. $\eta \varepsilon$ furnishes $z_{2}=-0.0003(7)$ as shown in Fig. 3(c) and (d), respectively. The crossover exponents $z_{1}$ and $z_{2}$ can be obtained also by using Eq. (18) and the previous values of $\alpha_{1}, \alpha_{2}$ and $\beta$. By doing so, we obtained $z_{1}=-0.98(1)$ and $z_{2}=0.00(1)$, which are in perfect agreement with the numerical data. A final check of the initial hypotheses can be seen in Fig. 2(c). As one can see in Fig. 2(c) all the curves overlap onto each other in a single and, hence, universal plot. Furthermore, the present result allows us to confirm that even in the stochastic dynamics, the introduction of dissipation is enough to suppress the unlimited energy growth. These results are confirmed by looking at Eqs. (15) and (16) and the previous values for the critical exponents $\alpha_{1}$ 
and $z_{1}$. Observe that both $\alpha_{1}$ and $z_{1}$ are negative, which lead to $\bar{V}_{\text {sat }} \propto 1 /(1-\gamma)^{\left|\alpha_{1}\right|}(\eta \varepsilon)^{\alpha_{2}}$ and $n_{x} \propto 1 /(1-\gamma)^{\left|z_{1}\right|}(\eta \varepsilon)^{z_{2}}$. Note that when $\gamma \rightarrow 1$, implies that $\bar{V}_{\text {sat }} \rightarrow \infty$ and $n_{x} \rightarrow \infty$, too, thus recovering the results for the conservative case leading then to Fermi acceleration. However, when $\gamma$ is slightly less than 1, it implies that the system has a characteristic saturation value $\bar{V}_{\text {sat }}$ and a crossover iteration number $n_{x}$.

\section{Analytical approach}

Since the results obtained in the previous section were purely numerical, a more detailed analytical investigation is needed. To study analytically the average velocity of an ensemble of noninteracting particles, the corresponding probability distribution for the velocity in the two-dimensional phase space, i.e., $\alpha$ vs. $\theta$, must be known. For the deterministic case, periodic islands are present in the phase space and obtain the probability distribution is a very difficult task when a time dependent perturbation is introduced. One possible way to overcome this limitation and to make the analytical work feasible is to assume that the probability distribution in the plane $\alpha v$ s. $\theta$ is uniform. This is exactly what happens when the stochastic term is introduced in Eq. (3).

Taking this situation into account, we can average Eq. (11) for the ranges $\theta \in[0,2 \pi], \alpha \in[0, \pi]$ and $t \in[0,2 \pi]$. After some algebra we obtain

$$
{\overline{V^{2}}}_{n+1}=\frac{{\overline{V^{2}}}_{n}}{2}+\frac{\gamma^{2} \bar{V}_{n}^{2}}{2}+\frac{(1+\gamma)^{2} \eta^{2} \varepsilon^{2}}{8} .
$$

This result will be used to discuss different regimes, namely, the stationary and the dynamical regime.

\subsection{Stationary state}

In the stationary regime the mean-squared velocity is obtained under the following condition $\overline{V^{2}}{ }_{n+1}=\overline{V_{n}^{2}}=\overline{V^{2}}$, and that when isolating $\overline{V^{2}}$ leads to

$$
\overline{V^{2}}=\frac{(1+\gamma) \eta^{2} \epsilon^{2}}{4(1-\gamma)}
$$

If we define the root mean square velocity as $\bar{V}=\sqrt{\overline{V^{2}}}$, we have

$$
\bar{V}=\frac{\eta \epsilon}{2} \sqrt{(1+\gamma)}(1-\gamma)^{-1 / 2} .
$$

From Eq. (21) we see the exponent heading the term $(1-\gamma)$ is $-1 / 2$ which is in perfect agreement with the exponent $\alpha_{1}$ obtained from Fig. 3(a). We notice also that $\alpha_{2}=1$, in agreement also with the result from the in-set of Fig. 3(a).

\subsection{Dynamical regime}

To investigate the behavior of the dynamical regime, the first thing we need to do is to transform a difference equation - relating $\bar{V}_{n+1}^{2}$ with $\overline{V_{n}^{2}}$ - from Eq. (19) into a differential equation whose solution is feasible. Then we assume that the average velocity, when averaged over a large ensemble, admits the following approximation

$$
{\overline{V^{2}}}_{n+1}-\overline{V_{n}^{2}}=\frac{{\overline{V^{2}}}_{n+1}-\overline{V_{n}^{2}}}{(n+1)-n} \cong \frac{\mathrm{d} \overline{V^{2}}}{\mathrm{~d} n},
$$

hence leading to

$$
\frac{\mathrm{d} \overline{V^{2}}}{\mathrm{~d} n}=\frac{\overline{V^{2}}}{2}\left(\gamma^{2}-1\right)+\frac{(1+\gamma)^{2} \eta^{2} \varepsilon^{2}}{8}
$$

Since this is a first order differential equation, it can be solved easily. Integrating the equation considering that at $n=0$ and initial velocity $V_{0}$ we obtain

$$
\overline{V^{2}}(n)=\overline{V_{0}^{2}} \mathrm{e}^{\frac{\left(\gamma^{2}-1\right)}{2} n}+\frac{(1+\gamma)}{4(1-\gamma)} \eta^{2} \varepsilon^{2}\left[1-\mathrm{e}^{\frac{\left(\gamma^{2}-1\right)}{2} n}\right] .
$$

When the initial velocity is sufficiently small, say $V_{0} \cong 0$, the dominant expression for $\overline{V^{2}}(n)$ is

$$
\sqrt{\overline{V^{2}}(n)}=\frac{(1+\gamma)^{1 / 2}}{2}(1-\gamma)^{-1 / 2} \eta \varepsilon\left[1-\mathrm{e}^{\frac{\left(\gamma^{2}-1\right)}{2} n}\right]^{1 / 2} \text {. }
$$


On the other hand, if the initial velocity is large enough, the leading expression shows and exponential decay given by

$$
\sqrt{\overline{V^{2}}(n)} \cong V_{0} \mathrm{e}^{\frac{\left(\gamma^{2}-1\right)}{4} n} \cong V_{0} \mathrm{e}^{\frac{(\gamma-1)}{2} n}
$$

Finally, the full dynamics of $\bar{V}(n)$ over an ensemble of initial condition is described by the following equation:

$$
\sqrt{\overline{V^{2}}(n)}=\sqrt{\overline{V_{0}^{2}} \mathrm{e}^{\frac{\left(\gamma^{2}-1\right)}{2} n}+\frac{(1+\gamma)}{4(1-\gamma)} \eta^{2} \varepsilon^{2}\left[1-\mathrm{e}^{\frac{\left(\gamma^{2}-1\right)}{2} n}\right]} .
$$

\subsection{Average over the orbit}

Since Eq. (27) gives us the average velocity over an ensemble of initial conditions, we need to make an average over the orbit (or the number of collision) to compare the results with Fig. 2. Therefore we define a new velocity written as

$$
V_{r m s}=\sqrt{\frac{1}{n+1} \sum_{j=0}^{n} \overline{V^{2}}(j)}
$$

The resulting velocity must be comparable with the one presented in Eq. (13). After doing some algebra we end up with

$$
V_{r m s}(n)=\sqrt{\frac{(1+\gamma)}{4(1-\gamma)} \eta^{2} \varepsilon^{2}+\frac{1}{(n+1)}\left[\overline{V_{0}^{2}}-\frac{(1+\gamma)}{4(1-\gamma)} \eta^{2} \varepsilon^{2}\right]\left[\frac{1-\mathrm{e}^{\frac{\left(\gamma^{2}-1\right)}{2}(n+1)}}{1-\mathrm{e}^{\frac{\left(\gamma^{2}-1\right)}{2}}}\right]}
$$

From the above result one can see that

$$
V_{r m s}=\left\{\begin{array}{l}
V_{0}, \quad \text { if } n=0 \\
\frac{\eta \varepsilon}{2} \sqrt{(1+\gamma)}(1-\gamma)^{-1 / 2}, \quad \text { if } n \rightarrow \infty .
\end{array}\right.
$$

The continuous line in Fig. 2(a) shows the behavior of the theoretical value of the square velocity (Eq. (29)) as a function of the number of collisions for different combinations of control parameters $\gamma$ and different values of $\eta \varepsilon$. As one can see, it reproduces remarkably well the results obtained numerically.

\subsection{Scaling exponents}

To obtain the scaling exponents one must consider three situations: (i) at the saturation, when $n \gg n_{x}, \bar{V}$ is described by Eq. (30) and after comparing it with Eq. (14) one can see that $\alpha_{1}=-1 / 2$ and $\alpha_{2}=1$. On the other hand, for small values of $n$ such as $n \ll n_{x}$, the mean-square velocity behaves according to Eq. (29). Assuming $V_{0} \ll 1$, and expanding the exponential in a Taylor series

$$
\mathrm{e}^{\frac{\left(\gamma^{2}-1\right)(n+1)}{2}}=1+\frac{\left(\gamma^{2}-1\right)(n+1)}{4}+\cdots
$$

and considering $(n+1) \cong n$ and taking only the lower order in the expansion, we can rewrite $\bar{V}$ as

$$
\bar{V}(n)=\frac{(1+\gamma)}{4}(\eta \varepsilon) n^{1 / 2} .
$$

Therefore, comparing Eq. (32) with Eq. (14) one can show that the acceleration exponent is $\beta=1 / 2$. Finally, the crossover iteration number $n_{x}$ that marks that change of behavior from growth to saturation can be estimated when Eq. (32) intersects Eq. (30). After some calculation we obtained

$$
n_{x}=\frac{4}{(1+\gamma)}(1-\gamma)^{-1} \text {. }
$$

After a comparison with the scaling hypotheses (16), we can show that the saturation exponents are $z_{1}=-1$ and $z_{2}=0$. The exponents $z_{1}$ and $z_{2}$ can be obtained also by using Eq. (18) and the previous values of $\beta, \alpha_{1}$ and $\alpha_{2}$.

\section{Connection with thermodynamics}

In this section, let us discuss a possible connection with the thermodynamic. To do so, we make use of the energy equipartition theorem [31,32] which can be given in a compact form as

$$
\frac{m}{2} \overline{V^{2}}=K_{B} T=U,
$$




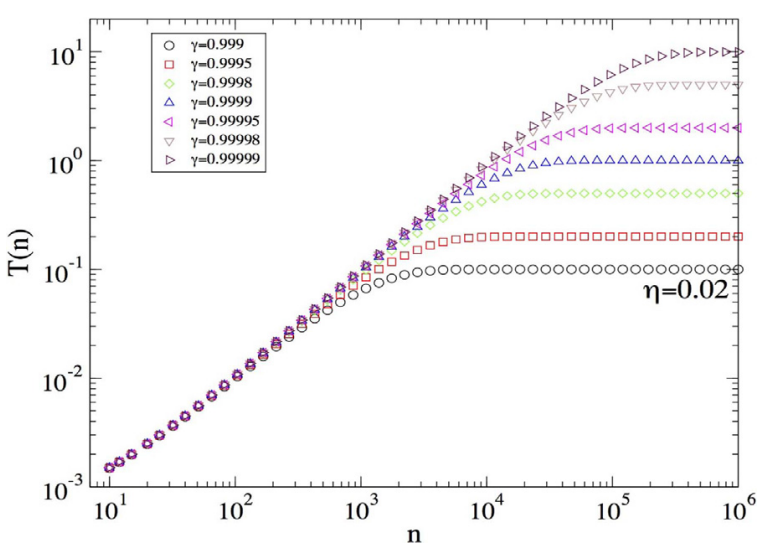

Fig. 4. Behavior of the temperature $T$ as a function of $n$.

where $K_{B}$ is the Boltzmann constant and $U$ is the energy of the gas of particles. Once the squared velocity is known, the temperature can be written as

$$
T(n)=T_{0} \mathrm{e}^{\left[\frac{\left(\gamma^{2}-1\right) n}{2}\right]}+\frac{m}{2 K_{B}} \frac{(1+\gamma)}{4(1-\gamma)} \eta^{2} \varepsilon^{2}\left[1-\mathrm{e}^{\left[\frac{\left(\gamma^{2}-1\right) n}{2}\right]}\right] .
$$

If the ensemble of particles in the billiard is started with low temperature $T_{0} \ll \frac{m}{2 K_{B}} \frac{(1+\gamma)}{4(1-\gamma)} \eta^{2} \varepsilon^{2}$, then the temperature will behave as in Fig. 4, exhibiting similar scaling features as seen in Fig. 2.

An expression for the entropy, as foreseen from statistical mechanics standpoint in differential form, then

$$
\mathrm{d} S=\frac{1}{T} \mathrm{~d} U
$$

Integrating this equation we end up with

$$
S=\tilde{S}+K_{b} \ln (U),
$$

where $\tilde{S}=S_{0}-K_{b} \ln \left(U_{0}\right), S_{0}$ is a constant and $U_{0}$ is the initial energy. We see that the entropy confirms a classical postulate from the thermodynamics [33] that it is a monotonically growing function of the energy.

\section{Conclusion}

As the concluding remark, we have studied some dynamical properties of a dissipative time dependent oval billiard. We introduced a random perturbation on the boundary and investigate the behavior of the average velocity of the particles as a function of the number of collisions for different combinations of the control parameters. We observed that average velocity grows for small number of collision and then, after a crossover, it reaches a regime of saturation for large $n$. Thus we do not observe the unlimited energy growth (Fermi acceleration). We have shown there is a relationship between the critical exponents $\beta, \alpha_{1}, \alpha_{2}, z_{1}$ and $z_{2}$. The exponents were used to show that the average squared velocity is scaling invariant with a perfect collapse of all curves onto a single universal plot. Additionally, we have given an analytical argument using the equilibrium condition at the steady state regime for the scaling exponents obtained numerically. Our procedure led to obtain the critical exponents $\beta=0.5, \alpha_{1}=-0.5, \alpha_{2}=1, z_{1}=-1$ and $z_{2}=0$ which are in perfect agreement with the exponents obtained numerically. The empirical expression for the average velocity allowed us to make a connection with the thermodynamic. Our results show that the average squared velocity - and hence the temperature - grows as a power law for $V_{0} \sim 0$, but and $n \rightarrow \infty$, it tends to a constant plateau. Furthermore, the weaker is the dissipation, the higher if the temperature in the steady state. Finally, we confirmed that the entropy is monotonically growing function of the energy.

\section{Acknowledgments}

MVCG acknowledges CNPq (303707/2015-1) for financial support. DFMO thanks to James S. McDonnell Foundation. EDL thanks FAPESP (2012/23688-5) and CNPq (303707/2015-1) for financial support.

\section{References}

[1] G. Birkhoff, Dynamical Systems, American Mathematical Society, Providence, RI, USA, 1927.

[2] N.S. Krylov, Works on the Foundations of Statistical Physics, Princeton University Press, Princeton, NJ, USA, 1979. 
[3] Ya.G. Sinai, Russian Math. Surveys 25 (1970) 137.

[4] L.A. Bunimovich, Funct. Anal. Appl. 8 (1974) 73.

[5] L.A. Bunimovich, Ya.G. Sinai, Comm. Math. Phys. 78 (1981) 479.

[6] N. Chernov, R. Markarian, Chaotic Billiards, vol. 127, American Mathematical Society, 2006.

[7] L.A. Bunimovich, Comm. Math. Phys. 65 (1979) 295.

[8] L.A. Bunimovich, Chaos 1 (1991) 187.

[9] L.A. Bunimovich, L.V. Vela-Arevalo, Chaos 22 (2012) 026103.

[10] A.B. Ryabov, A. Loskutov, J. Phys. A 43 (2010) 125104.

[11] E. Fermi, Phys. Rev. 75 (1949) 1169.

[12] A. Loskutov, A.B. Ryabov, L.G. Akinshin, J. Phys. A 33 (2000) 7973.

[13] A. Loskutov, A.B. Ryabov, L.G. Akinshin, J. Exp. Theor. Phys. 89 (1999) 966.

[14] D.F.M. Oliveira, J. Vollmer, E.D. Leonel, Physica D 240 (2011) 389.

[15] A.K. Karlis, P.K. Papachristou, F.K. Diakonos, V. Constantoudis, P. Schmelcher, Phys. Rev. E 76 (2007) 016214.

[16] E.D. Leonel, D.F.M. Oliveira, A. Loskutov, Chaos 19 (2009) 033142.

[17] C.P. Dettmann, O. Georgiou, Phys. Rev. E 83 (2011) 036212.

[18] A.L.P. Livorati, A. Loskutov, E.D. Leonel, Physica A 391 (2012) 4756.

[19] V. Gelfreich, D. Turaev, J. Phys. A 41 (2008) 212003.

[20] V. Gelfreich, D. Turaev, Comm. Math. Phys. 283 (2008) 769.

[21] M.V. Berry, European J. Phys. 2 (1981) 91.

[22] F. Lenz, F.K. Diakonos, P. Schmelcher, Phys. Rev. Lett. 100 (2008) 014103.

[23] F. Lenz, C. Petri, F.R.N. Koch, F.K. Diakonos, P. Schmelcher, New J. Phys. 11 (2009) 083035.

[24] F. Lenz, C. Petri, F.K. Diakonos, P. Schmelcher, Phys. Rev. E 82 (2010) 016206.

[25] E.D. Leonel, L.A. Bunimovich, Phys. Rev. Lett. 104 (2010) 224101.

[26] D.F.M. Oliveira, E.D. Leonel, Physica A 389 (2010) 1009.

[27] A.L.P. Livorati, I.L. Caldas, E.D. Leonel, Chaos 22 (2012) 026122.

[28] D.F.M. Oliveira, M. Robnik, Phys. Rev. E 83 (2011) 026202.

[29] E.D. Leonel, A.L.P. Livorati, Commun. Nonlinear Sci. Numer. Simul. 20 (2015) 159.

[30] A. Loskutov, Phys. Usp. 50 (2007) 939.

[31] F. Reif, Fundamentals of Statistical and Thermal Physics, McGraw-Hill, New York, 1965.

[32] K. Huang, Statistical Mechanics, John Wiley \& Sons, New York, 1963.

[33] H.B. Callen, Thermodynamics and an Introduction to Thermostatistics, second ed., John Wiley \& Sons, New York, 1985. 